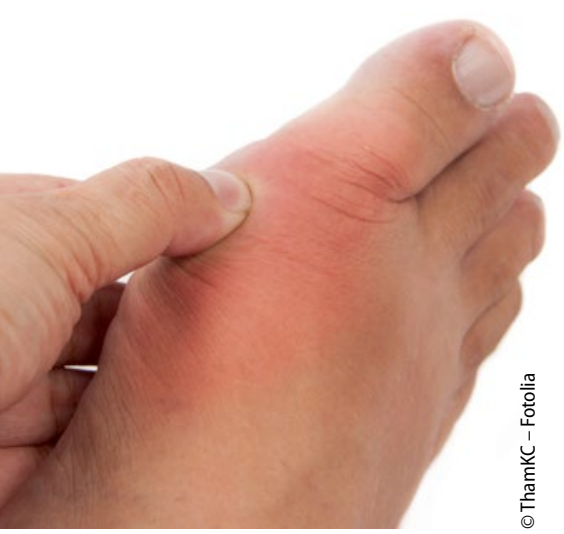

Monarthritis des Großzehengrundgelenks - dieser "Klassiker" ist bei Gicht nicht die Regel.

Mehr als „nur" ein Gelenkproblem

\title{
Gicht: Systemerkrankung mit vielen Facetten
}

\author{
Die Gicht hat in den letzten Jahren eine erstaunliche Karriere von \\ einer monolokulären Gelenkerkrankung zu einer systemischen \\ autoinflammatorischen Erkrankung gemacht. Für die Praxis besonders \\ wichtig: Das klinische Bild der Erkrankung ist keineswegs immer so \\ klassisch wie es die meisten kennen.
}

Lage, überschießend zu reagieren. Dann stehen weniger die klassischen akuten Gelenksymptome im Mittelpunkt, sondern es entwickeln sich frühzeitig Tophi und eine chronische Arthritis.

Auch bei chronischen muskuloskeletalen Beschwerden sollte man durchaus auch an eine chronische Gicht denken. „Suchen Sie nach Tophi bei Patienten, die immer wieder mit unklaren Bursitiden kommen“, riet Reuss-Borst. Auch unklare Tendovaginitiden können Ausdruck einer chronischen Gicht sein. Verdächtig ist auch, wenn jüngere Patienten Arthrosen entwickeln.

Da Harnsäure-Kristall-Ablagerungen auch an ungewöhnlichen Lokalisationen heute gut nachweisbar sind, kann man immer wieder Überraschungen erleben, wo sich Gichttophi bilden, sagte Reuss-Borst. Sie erwähnte z.B. den Riesenzelltumor oder unklare Nagelveränderungen, die sich als Gichttophi entpuppten, oder Tophi an der Wirbelsäule als Ursachen von Rückenschmerzen oder gar eines Querschnittsyndroms.

Auch der Gelenkultraschall hat dazu beigetragen, dass der Facettenreichtum der Gicht heute klarer geworden ist. Man findet die typischen sonografischen Veränderungen, echoinhomogene wolkige Doppelkonturen, nicht nur in Gelenken, die klinisch manifest von der Gicht betroffen sind, sondern auch in asymptomatischen Gelenken.

\section{Dr. med. Angelika Bischoff}

- 121. Kongress der Deutschen Gesellschaft für Innere Medizin e.V., Mannheim 2015

\section{Gicht und Arthrose unterstützen sich gegenseitig}

Gichtanfälle treten mit achtfach höherer Wahrscheinlichkeit in Arthrose-vorgeschädigten Gelenken auf. Denn bei fortschreitender Arthrose werden Zellen im Gelenk apoptotisch, und es fällt Harnsäure an, die auskristallisiert und das Inflammasom aktiviert. Es wird Interleukin1-beta freigesetzt. Da das Großzehen-Grundgelenk ein Prädilektionsort der Arthrose ist, tritt genau dort oft auch der erste Gichtanfall auf. Durch die niederschwellige Entzündung fördert die Gicht umgekehrt das Fortschreiten der Arthrose.

\section{Gicht schadet dem Herz-Kreislauf-System}

Ein ständig aktiviertes Inflammasom hat auch negative Auswirkungen auf Herz, Gefäße und Nieren. Im Tiermodell konnte gezeigt werden, dass Harnsäure zu einer Freisetzung von proinflammatorischen Zytokinen in der Gefäßwand führt. Nicht nur mit einer manifesten Gicht, sondern auch einer asymptomatischen Hyperurikämie geht ein deutlich erhöhtes kardiovaskuläres Risiko einher. Eine Niereninsuffizienz lässt auf der einen Seite den Harnsäurespiegel im Blut ansteigen. Auf der anderen Seite bewirkt die Hyperurikämie, dass die Niereninsuffizienz rascher voranschreitet. Behandelt man die Patienten mit Allopurinol, lässt sich auch die glomeruläre Filtrationsrate stabilisieren. 